\title{
Information conception of image perception at solid-state lighting
}

\author{
V.I. Osinsky \\ Institute of Microdevices, Kyiv, Ukraine; e-mail: osinsky@imd.org.ua
}

\begin{abstract}
An information-technological approach to the development of integral white light sources is introduced. It is based on the technology of epitaxial growth of nanostructures in multicomponent solid-state solutions of $\mathrm{A}^{3} \mathrm{~B}^{5}$ compounds and their combination with silicium-based microdevices. We present a new conception of the perception of patterns by man's eye analyzer. It consists in the registration and the processing of biograms and neurograms which are analogs of optical holograms. It is shown that solid-state lighting allows one to perceive information which is equivalent to an energy efficiency of $150-200 \mathrm{Lm} / \mathrm{W}$. This is economically profitable when going to solid-state lighting.
\end{abstract}

Keywords: image perception, solid-state lighting, biogram, neurogram, $\mathrm{A}^{3} \mathrm{~B}^{5}$ compound.

Manuscript received 12.07.07; accepted for publication 27.09.07; published online 31.10.07.

\section{Introduction}

Unlike lamps, solid-state light (SSL) sources are not powerful, but information-bearing elements, completely compatible by all physico-technological parameters with microelectronic processors, sensors of light beams, microoptics, power suppliers, and switches of lightemitting diodes (LEDs) which are solid-state sources of illumination (Fig. 1) [1].

These micro- and optoelectronic elements make measurement of the parameters of external illumination and distributions of brightness and colors, filtration, and separation of contours. In this case, the algorithm of the switching-on of a multispectral multilayered LED structure of the RGB type that reduces the energy expenses for illumination can be optimized.

In comparison with a continuous spectrum of lamps, each LED is a monochromatic source of radiation that promotes the interference, coding, and filtration. The optimization of perception of the information can be made by the program of a microprocessor or a man during the work of a source of illumination. Manufacturing the SSL devices is realized by the hybrid or monolithic technology with the use of methods of integration of elements on silicon and multicomponent solid-state solutions of $\mathrm{A}^{3} \mathrm{~B}^{5}[2]$.

\section{Macro- and micropeculiarities of visual registration of images}

The important properties of man's visual system for the perception of images at SSL are: the inertia concerning the duration of the switching of LEDs, adaptability, ability to generalize and to identify images, a wide range of registered illumination intensities which expands over 9 orders, the ability to register the brightnesses in one image (a dynamic range of brightness) which differ by about 3 orders, and the high visual acuity (spatial resolution).

The problem of perception of images at LED-based illumination is usually considered on the basis of the information display systems developed in TV and robotics and methods of the theory of circuits which considers one-dimensional signals.

In fact, the processing of images in the visual analyzer of a man is carried on both consistently and in parallel with the simultaneous processing of all elements of the image. In comparison with other sources of illumination, superbright LEDs based on quantumdimension heterostructures radiate narrow spectral strips which possess a rather high coherence degree, in which not only the amplitude is essential, but also the phase of a light wave, $A(x, y) \cdot e^{i \varphi(x, y)}$. In such an interpretation, it is necessary to consider the interference of light waves in optical parts of a visual analyzer, as a result of which interferograms-holograms are formed. The higher the coherence degree of light waves of illumination, the less the loss of radiation in the formation of qualitative holograms. Ideal coherent sources are lasers. Partially coherent radiation of LEDs is capable to form holograms with smaller losses of radiation in comparison with all other applied sources of illumination.

The image of a subject in the analog form acts on the optical system of eye, in which it is focused on retina, where its digitization, transformation of the optical signal into an electric one, preliminary processing, and transfer along nervous fibers into brain occur (Fig. 2) [3]. 
SSL Source

Human sight analyzer

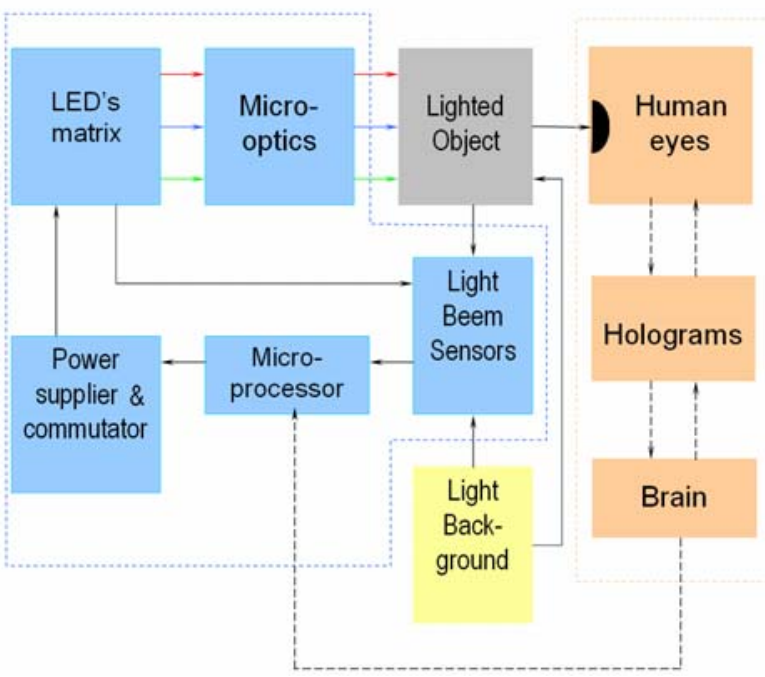

Fig. 1. Functional scheme of the effect of an SSL source on man's sight analyzer.
A two-dimensional (2D) or three-dimensional (3D) image is focused by a crystalline lens on photosensitive cells of retina [retinal rods and cones (Fig. 2)], in the entrance plane of which interferograms-holograms are formed. About 125 million rods are positioned on the whole hemispherical surface of retina and realize blackand-white twilight sight. In the central area of retina are located about 6.5 million cones which register the color scale of subjects at their bright illumination. Photosensitive cells are located in a back layer of retina (Fig. 2a). Light falls on them after the passage of several layers of nervous cells. Rods and cones are oriented to light by their internal segment which does not contain a visual pigment. As nervous cells and internal segments are transparent for visible light, they absorb a very small part of the light flux. In them, there occurs the "coherentization" of the light flux (Fig. 2b).

The sight sensitivity threshold of a man after the adaptation in full dark is equal to $(2.1-5.7) 10^{-17} \mathrm{~J}$ on the cornea surface of eye, which corresponds to $58-148$ quanta of blue-green light. From them, only 5-15 quanta
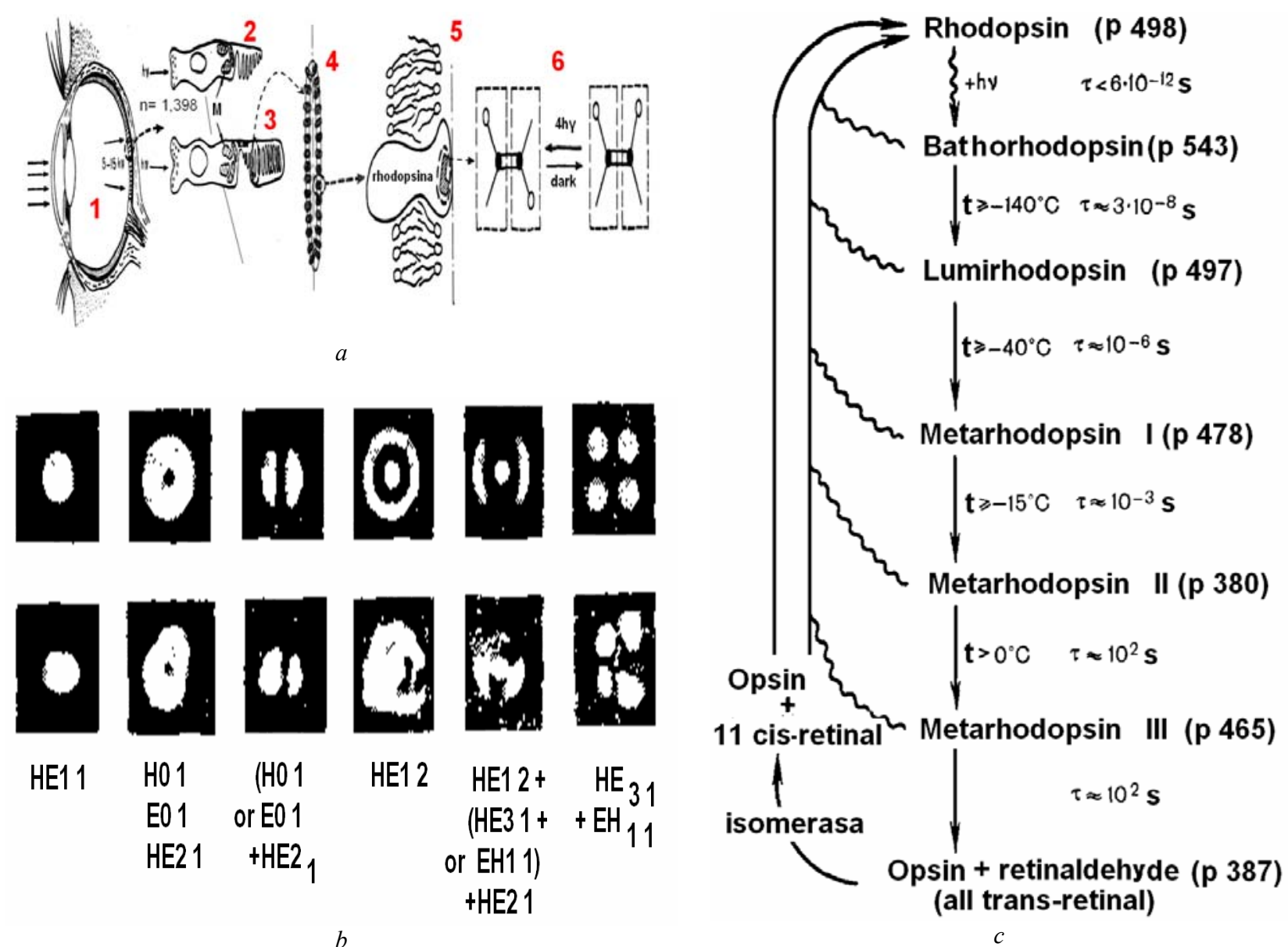

$b$

Fig. 2. The structure of sight organs: a) 1 - cross-section of eye, 2 - cones, 3 - rods, 4 - ellipsoid, 5 - rhodopsin, 6 - cis-transisomerization of retinal under the action of light irradiation; $b$ ) photos of the distribution of various modes on the receptors of human retina (lower series) and ideal pictures on the upper series, $\mathbf{H}$ and $\mathbf{E}$ - magnetic and electric vectors; $c$ ) conformational transformations of rhodopsin under the action of light irradiation. 
are absorbed by molecules of the visual pigment rhodopsin which is built in the disks which are lipid membranes laid in a pile. A congestion of mitochondria is located between the internal and external segments of rods and cones. At the end of an internal segment, there is a contact with a nervous fiber. Molecules of a pigment can quickly diffuse in a liquid lipid layer. After the absorption of a quantum of light, the conformational reorganizations consisting in the photoisomerization of retinal, being a component of rhodopsin occur (Fig. 2c). Photoisomerization is a reversible reaction that causes both the registration of light and the regeneration of a visual pigment. Conformation reorganizations of rhodopsin were first investigated by G. Wald who has received the Nobel Prize for his work in 1968. Six derivatives of rhodopsin were revealed. Their lifetimes decrease from $10^{2} \mathrm{~s}$ up to $10^{-12} \mathrm{~s}$ (Fig. 2c) with increase in temperature from $-140^{\circ} \mathrm{C}$ up to room one. Regeneration of rhodopsin in darkness occurs under the action of enzymes.

\section{Partial coherence of superluminescent LEDs}

The superluminescence represents the electroluminescence mode of LEDs, in which there is a high enough degree of inversion of population densities in the conduction band of the active layer of a double heterostructure (Fig. 3) with the optical and electronic restriction of light and carriers of the current. Wide-band layers of the $n$ - and $p$-types have smaller refractive indices than that of the active layer with narrower band. For vertically propagating quanta of light, this creates a Fabry-Perot resonator, in which the induced radiation of light happens. That is, we deal with the optical amplification of light, a prelaser mode, which does not pass in the generation because of an insufficient positive feedback at such currents and the geometry of a structure (Fig. 3). However, the degree of coherence of the electromagnetic field essentially increases, which leads to the increase in both the informational capabilities of the radiation of LEDs and its perception by the visual apparatus of a man.

The consequence of an increase in the share of the induced radiation is the narrowing of a spectral line, enhancement of the orientation of radiation along a direction perpendicular to mirrors of the resonator, and decrease in the delay time of optical radiation relative to the electronic excitation. We observed these effects in experiments in heterogeneous radiating $p$ - $n$ structures in multicomponent solid-state solutions of arsenide-phosphide of gallium-indium in the superluminescent mode (Fig. 4) which transits into the laser mode of coherent radiation at the achievement of the threshold current [2, 4].

The mode of a superluminescence in semiconductor structures is used in amplifiers of light, in which a structure similar to the surface emitting laser with a vertical resonator [5] is optimum. In our case (Fig. 4), the current pulses of a duration of $0.1-1$ ns were
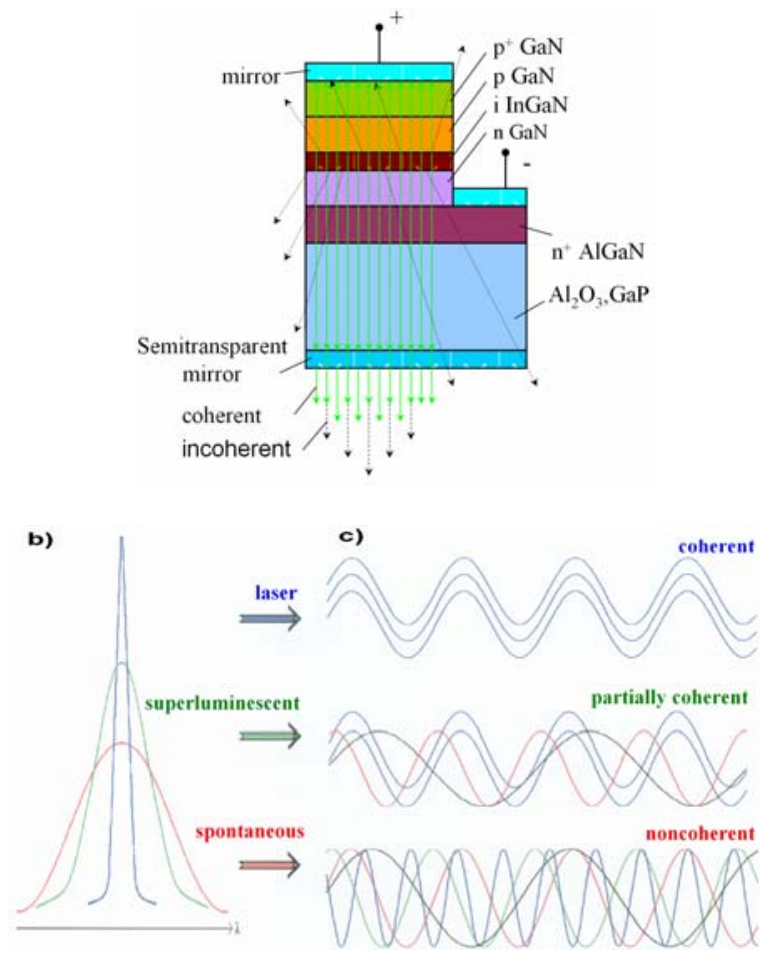

Fig. 3. $a$-structure of a superluminescence chip; $b$-spontaneous, superluminescent, and laser modes of a superluminescent LED, and the corresponding degree of coherence (c).
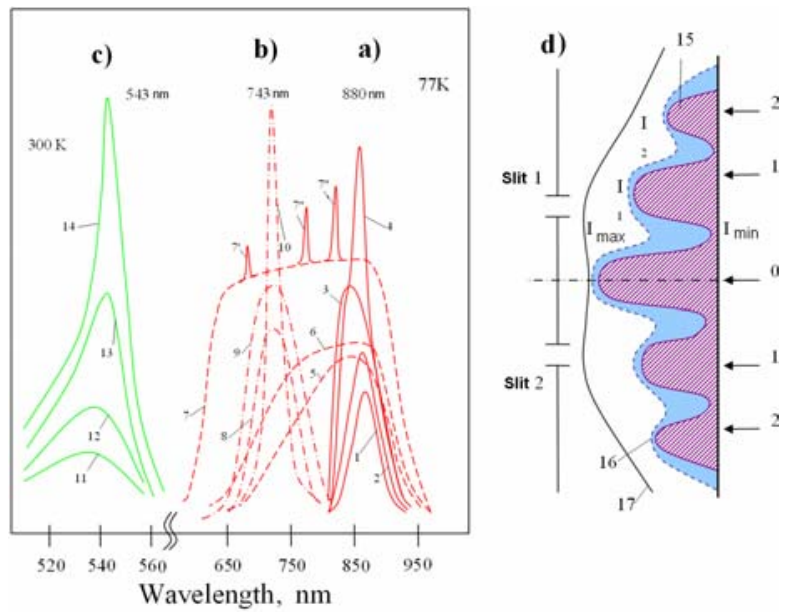

Fig. 4. Spectra of heterogeneous LEDs. $a$ ) $p$ - $n$ junction in $(\mathrm{InP})_{0.9}(\mathrm{GaAs})_{0.1}: 1,2$ - spontaneous luminescence, 3 superluminescence, 4 - stimulated emission; $b$ ) heterogeneous solid-state solutions: $5-(\mathrm{InP})_{0.3}(\mathrm{GaAs})_{0.7}, 6-$ $(\mathrm{InP})_{0.4}(\mathrm{GaAs})_{0.6}, 7-(\mathrm{InP})_{0.5}(\mathrm{GaAs})_{0.5}, 8,9$ - photoluminescence, 10 - laser emission of the macroheterogeneous composition $(\mathrm{InP})_{0.3}(\mathrm{GaAs})$ at pulse excitation by a nitrogen laser; c) 11,12 - spontaneous radiation, 13 - superluminescence, 14 - laser emission of the heterostructure $\mathrm{GaN} / \mathrm{InGaN} / \mathrm{GaN} / \mathrm{Al}_{2} \mathrm{O}_{3}$ at a current density of $70 \mathrm{~A} / \mathrm{cm}^{2}$ (11), $80 \mathrm{~A} / \mathrm{cm}^{2}$ (12), $100 \mathrm{~A} / \mathrm{cm}^{2}$ (13), $200 \mathrm{~A} / \mathrm{cm}^{2}$ (14); d) experimental setup to measure the degree of coherence $\gamma$ by analyzing the two-hole interference: 15 - for a heterolaser, 16 - for a superluminescent LED in a partially coherent mode, 17 - for a spontaneous LED. 
applied for the excitation of the superluminescent mode. The superluminescent mode appears at a current of $1.5 \mathrm{~A}$ with a pulse duration of $1 \mathrm{~ns}$, that corresponds to a current density of $600 \mathrm{~A} / \mathrm{cm}^{2}$ through the chip of $0.5 \times 0.5 \mathrm{~mm}$ in size. At smaller durations of pulses, the inversion of occupation densities happens at smaller densities of the current. For heterostructures of the fourcomponent InGaAsP system at a duration of pulses of $200 \mathrm{ps}$, superluminescent radiation was observed at densities of currents near $100 \mathrm{~A} / \mathrm{cm}^{2}$. For nitride-based heterostructures, this value is approximately twice more.

\section{Holographic model of sight}

In the theory and practice of holography, the methods of holographing without a reference beam [7] are developed. They are based on the registration of a change of the phase of a light wave by an object. The threedimensional object makes a phase modulation of light waves. The connection between a shift of the phase and the light field amplitude is actually registered. Thus, the separation of optical signals against of the noise background is easily realized. In Fig. 5, the scheme of holographing without a reference beam is shown under the illumination of object 3 through medium 2 by lightemitting diode 1 . The reflected wave comes through the medium to eye's crystalline lens 4 . Schemes of holographing without a reference beam are realized twice after the lenses of crystalline lens 4 and ellipsoid 6 (Fig. 5). At the focus of crystalline lens 4 , the receptors of rods and cones are located. The distribution of a field on the input of eye's crystalline lens $u(x, y)$ is connected with field $U(u, v)$ in a focal plane of the lens by the Fourier-transformation

$U(u, v)=C_{1} F\{U(x, y)\}$, where $F$ is the designation of the operation of Fouriertransformation over spatial frequencies $u=k x / f$, $v=k y / f ; f$ is the focal length, and $C_{1}$ is a constant. The distribution of the intensity of light waves in the focal planes of registration 5 and 7 is determined as

$\Phi_{1}(u, v)=C_{2} U(u, v) U^{*}(u, v)$,

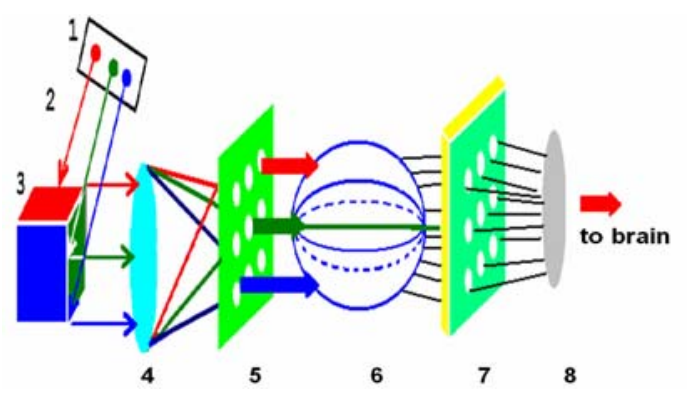

Fig. 5. Holographing without reference beam in man's sight analyzer: 1 - superluminescent LED's matrix, 2 - white (RGB) light beam passing though the medium, $3-3 \mathrm{D}$ object of lighting, 4 - crystalline lens, 5 - gradans of the fiber optics of eye's retina, 6 - ellipsoid, 7 - rhodopsin, 8 - neurons going to brain. where $U^{*}$ is the conjugate wave. The amplitudes of waves at each point of the planes of registration are determined by the sum of the amplitudes taken with the phase from every point of the object. That is, the onebeam hologram (diffractogram) by itself carries the full information on the amplitude and the phase of a light wave scattered by the object.

Transparent nervous fibers represent optical wave guides with the indices of refraction $n=1.398$ for an internal vein and $n=1.361$ for the environment medium. They are left with bunches of light with a high degree of coherence of photons, which is testified by photos of the distribution of intensities for the different modes obtained in monochromatic light on the terminations of receptors (Fig. 2c). Before the plane of receptors, densely packed oriented mitochondria form an ellipsoid. Long axes of mitochondria are positioned in parallel to the membrane of a cell and its longitudinal axis. The index of refraction of the environment ellipsoid is equal to $1.39-1.398$, which is essentially higher than that of the intercellular environment-myoid which lies between 1.334-1.347. In the process of passage of light from an internal segment through the ellipsoid to an external segment, the index of refraction increases, but it is always higher than that of the intercellular environment. In cones and rods at the transition from one area of a cross-section to another one, no jump-like change of the index of refraction occurs. These changes occur more or less smoothly. Actually, these wave guides are the gradient fibers similar to quartz gradient fibers gradans. For the parabolic profile of change of the index of refraction, a light beam is focused similar to the case of a lens. It is possible to strictly show that the selection of modes is realized on the output, and a partially coherent radiation goes out from the extended wave guide. Superposition of such beams gives the interference picture-hologram.

Partial coherence of light waves causes both a decrease in the clearness of interference pictures and the step-type behavior of light beams forming the hologram. Actually registered holograms are made of the set of elementary microholograms formed by mutually correlated "spots" of the objective and reference beams [7]. Investigations on quartz multimode fibers lighted by superluminescent radiation of LEDs show that the sizes of elementary microholograms correspond to the sizes of these spots and depend on the mode structure of optical guides (Fig. 2). In this case, the spectrum of registered spatial frequencies is narrowed; however, the details of images are kept, because the fields on neighboring spots correlate with those on the remote elements of holograms. The image restored from such holograms represents a result of addition of the images restored from all elementary microholograms. Therefore, details in the image are kept, but they are blurred. This leads to the deterioration of the discernability of especially fine structures. The ellipsoid is actually an optical convex lens, in the focal plane of which the Fourier-image of the light field of a considered object is formed. That part of 
radiation which passes by the ellipsoid represents a basic beam, whose interference with the objective wave creates holograms in the focal plane of the ellipsoid. This plane contains molecules of rhodopsin, in which holograms are registered. That is, the set of light bunches coherentized in nervous wave guides interferes with reference signals or among itself in the optical part, by forming a matrix of holograms-biograms in the layer of rhodopsin molecules. Optical holograms in molecules of rhodopsin will be transformed into the corresponding distributions of potentials at the terminations of nervous fibers (neurons). For convenience of the analysis, we name a potential biogram as a neurogram. In fact, there happens a synthesis of biograms-neurograms in the potential links of nervous terminations, along which signals are transferred into brain. Receptor potentials are a result of conformation transformations of rhodopsin under the action of light. After the illumination of a rod by a short flash, the early receptor potential (ERP) with a duration of about $1 \mathrm{~ms}$ is observed. Its amplitude increases with the intensity of a flash, but does not exceed $5 \mathrm{mV}$. Then, approximately in $1 \mathrm{~ms}$, the late receptor potential (LRP) is developed. It is considered that ERP is caused by conformation transformations, whereas LRP is caused by the movement of ions of sodium and potassium. For this time, sensors and a microprocessor can make the corresponding corrections of a current through LEDs (Fig. 6).

The maxima of sensitivity of the eye of a man are located nearby 500 and $550 \mathrm{~nm}$ under weak and strong illuminations, respectively. This is determined by absorption in rods and cones of rhodopsin which is called in the latter case as iodopsin. The maxima of absorption of man's iodopsin are located at 445, 535, and $570 \mathrm{~nm}$.

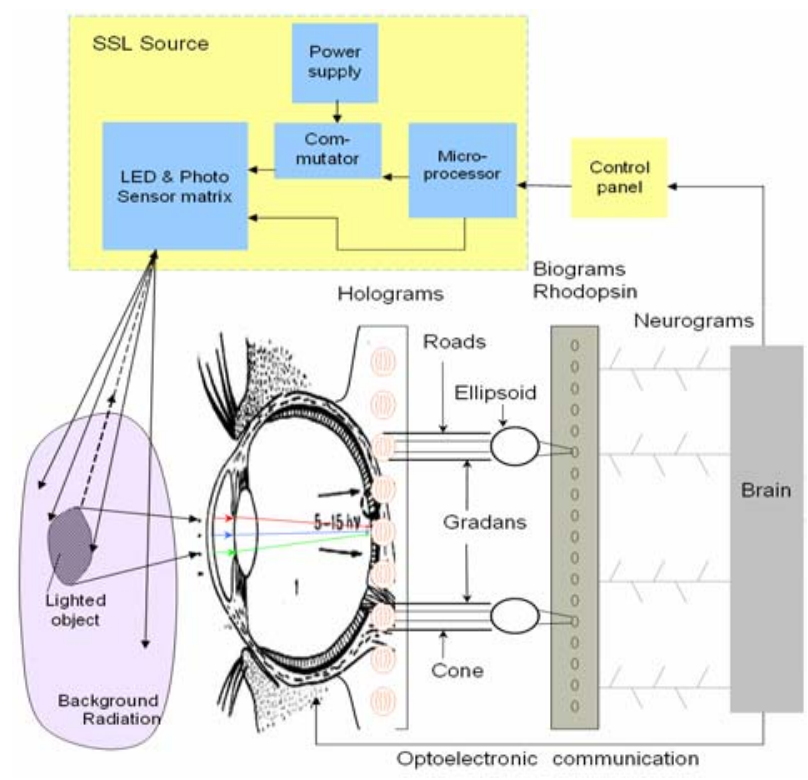

Fig. 6. Optoelectronic model of image registration at SSL lighting.

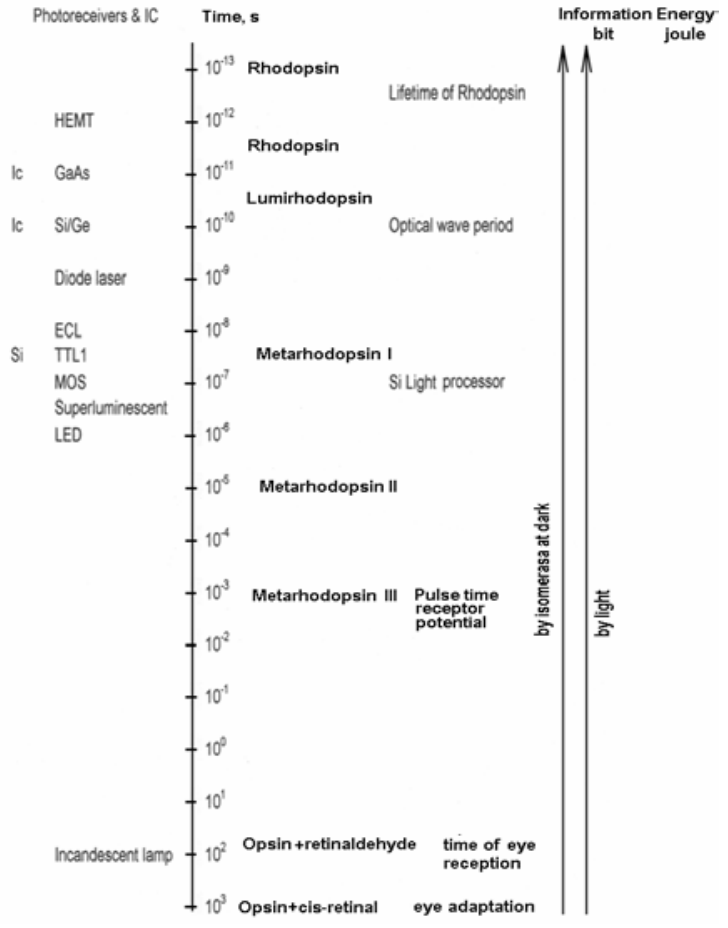

Fig. 7. Time parameters of sight and optoelectronic devices for their registrations.

In brain, the signals of transmitting neurons change the electric state of the dipoles of neurons of cerebral cortex which are distributed uniformly and their dipole moments are perpendicular to the cerebral cortex surface. Their activity is partially correlated, and their fields are added. The total (difference) value of potential underlies the mechanism of recognition of images by man's visual analyzer. We note also that images are also the distributions of receptor potentials from all sense organs: speech, music, smells, etc.

\section{Dynamical registration of images}

High speed of LED elements allows one to apply the dynamic registration of the information which separates the helpful information from the signals perceived by a man and the microprocessor with optoelectronic scheme of their interaction (Figs. 1, 6, and 7).

The changes in the images of subjects are separated and added to the initial information. However, the initial redundancy remains. To decrease it, one uses the system of sensors, a reduction of the sizes of the illumination field, a change of the spatial position of the optical axis, and changes of the scale of an image, color scale, exposition, and frequency of shots. For a further decrease of the redundancy of information, it is expedient to perform the preliminary processing of images. For a quite imperceptible time, the microprocessor can measure the parameters of external illumination, a filtration, can separate contours, and determine the distributions of brightness and colors. 
These data allow one to optimize the algorithm of switching-on of RGB of LEDs, which will reduce the expenses of energy for illumination.

The classical idea of the transformations of signals in the visual system includes parts of the logic restriction of levels of brightness and a Ziegert-Kotelnikov receiver which contains the subtracting, squaring, and averaging parts (Fig. 8) [8]. In a receiver, there occur a restriction of the dynamic range and the comparison of a neural image to the image stored in the memory of the observer. Inclusion of the concept of formation of images is caused by the logic of the analysis. Otherwise, it would be necessary to admit that a man stores their images in various foreshortenings, turns, scales, etc. in the memory in order to recognize the surrounding objects. The simple calculation shows that the memory size necessary for it exceeds considerably the total number of neurons of brain and spinal cord which is equal $13 \cdot 10^{10}$, and the number of connections between neurons is equal to $(3.9-6.5) \cdot 10^{12}$. We note that the number of neurons of a visual nerve is equal to $(0.8-1) \cdot 10^{6}$, which is less by five orders than that in the central nervous system. Spectral parameters of man's visual analyzer are given in Table 1.

As the number of visual neurons is 8 times less than that of cones and 150 times less than the number of rods, each neuron registers the potential from 8 and 150 subholograms, respectively. Using the habitual terminology, it is possible to present the primary holograms by analogs of words, from which pages of the information are formed. The distribution of the potentials of pages is transferred to brain where their comparison among themselves and with the distributions of potentials stored in the memory is realized.

According to the Kotelnikov-Shannon theorem, it is sufficient to calculate values of the Fourier-image at the chosen points for the calculation and storage of the spectrum of a signal. This allows one to reduce the volumes of necessary memory and time of processing. In the last case, a fast Fourier transformation is applied. The formation of holograms-biograms-neurograms is the essential mechanism of reduction of the volume of calculations.

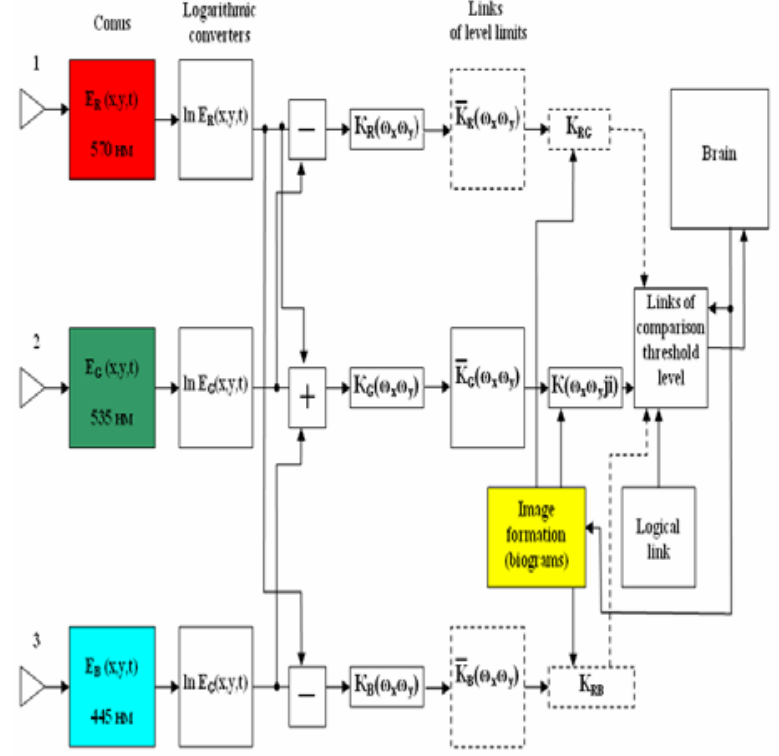

Fig. 8. Consequent radiotechnical model of man's sight analyzer.

The distribution of the complex amplitudes of a light wave in the plane of registration of holograms $x_{h}, y_{h}$ is proportional to the Fourier-image of an input signal

$$
\begin{aligned}
& U_{h}\left(x_{h}, y_{h}\right)= \\
& =\frac{A}{i \lambda f} \int_{-\infty}^{+\infty} U_{1}\left(x_{1}, y_{1}\right) \exp \left[-\frac{2 \pi i}{\lambda f}\left(x_{h} x_{1}+y_{h} y_{1}\right)\right] d x_{1} d y_{1} .
\end{aligned}
$$

Here, $x_{1}, y_{1}$ are the coordinates in the plane of input data, $A$ is the amplitude of an objective light wave, and $x_{h}, y_{h}$ are the coordinates in the plane of a hologram.

On the input, we have $M_{1} \times N_{1}$ bits of the information. Having executed the Fourier-transformation of expression (1), we find the density of registration of the digital information in the Fourier-hologram with area $S_{h}$ :

$$
n=\frac{M_{1} N_{1}}{S_{h}}=\frac{4 M_{1} N_{1}}{\pi d_{h}^{2}} \approx \frac{0.214 \alpha_{1} d_{1}^{2} M_{1}^{2}}{(\lambda f)^{2}},
$$

Table 1. The compositions of layers of the system InGaAINAsP which radiate light corresponding to the maxima of the

\begin{tabular}{|c|c|c|c|c|c|c|c|c|c|}
\hline Number & $\lambda_{\max }, \mathrm{nm}$ & $E_{\mathrm{g}}, \mathrm{eV}$ & $a, \mathrm{~nm}$ & In & $\mathrm{Ga}$ & $\mathrm{Al}$ & $\mathrm{N}$ & As & $\mathrm{P}$ \\
\hline 1 & 445 & 2.76 & 0.5195 & 0.0176 & 0.620 & 0.3625 & 0.345 & 0.405 & 0.265 \\
\hline 2 & 535 & 2.29 & 0.543 & 0.050 & 0.651 & 0.360 & 0.141 & 0.522 & 0.340 \\
\hline 3 & 570 & 2.15 & 0.5480 & 0.0085 & 0.675 & 0.316 & 0.100 & 0.535 & 0.365 \\
\hline 4 & 500 (rods) & 2.46 & 0.5352 & 0.0045 & 0.635 & 0.3605 & 0.170 & 0.51 & 0.332 \\
\hline 5 & 555 (cones) & 2.236 & 0.5402 & 0.0251 & 0.650 & 0.3252 & 0.130 & 0.521 & 0.352 \\
\hline 6 & $\mathrm{GaN}$ & 3.44 & 0.4503 & & & & & & \\
\hline 7 & $\begin{array}{c}\mathrm{Al}_{2} \mathrm{O}_{3} \text { (sapphire) } \\
(0001)\end{array}$ & & 0.4103 & & & & & & \\
\hline 8 & $\mathrm{SiC}$ & 3.25 & 0.307 & & & & & & \\
\hline 9 & $\mathrm{GaP}$ & 2.27 & 0.545 & & & & & & \\
\hline 10 & $\mathrm{ZnO}$ & 3.37 & 0.3245 & & & & & & \\
\hline
\end{tabular}
sensitivity of rhodopsin of cones (iodopsin) $(1,2,3)$ and the sensitivity of eye under weak (4) and strong (5) illumination; (6-10) - parameters of substrates for the epitaxial growth of III-nitrides. 
where $\alpha=N_{1} / M_{1}$; for the square input page, $M_{1}=N_{1}$ and $\alpha=1 ; d_{1}$ - diameter of a light beam on the input of an ellipsoid which is equal to the diameter of the optical wave guide of a fiber.

The diameter of a hologram in rhodopsin

$$
d_{h}=2.44 \beta_{1}(\lambda F) M_{1} \sqrt{1+d_{1}^{2}},
$$

where $\beta_{1}$ is the relative distance between fibers, $F=$ $f / D_{\mathrm{FL}}$ - aperture number of a lens with effective range $D_{\mathrm{FL}}$. Typical values of the parameters entering into formulas (2) and (3) are $\beta_{1} \geq 2$ and $F \geq 1$. For a red LED at $M_{1}=128, n=1 \cdot 10^{5} \mathrm{bits} / \mathrm{mm}^{2}$ and $d_{h}=0.4 \mathrm{~mm}$.

As well as in the case of Fourier-holograms obtained by means of a laser and a header, a biogram possesses high reliability of registration of the information, at which the loss of a part of the spectrum does not lead to the loss of the whole image. In biograms, the space-frequency filtration that is especially important for recognition and identification of the images based on the associative properties of biograms is made. These properties are important for the fast search of the information by semantic attributes which are contained in "keywords" of the image of an object (contour, color). The information on an object is registered not in the form of a direct image, but in the form of an interference picture - a biogram which is transferred in the form of neurograms to brain, where the spectrum of spatial frequencies of an input signal is actually registered. In this case, the high noise stability inherent in Fourier-holograms is realized. Visual Fourier-holograms-biograms possess all known advantages of usual optical Fourier-holograms: the invariance to shifts, high density of storage of the information, small aberrations, space-frequency filtration, associativity of the identification of images on the basis of the autocorrelation principle (autocorrelation function).

\section{Multispectral diode-based light processors (LP)}

Perspective substances for the creation of nanostructures in the whole range of the visible spectrum are sixcomponent solid-state solutions in the system AlGaInNPAs, in which the energy gap can change from 0.36 (InAs) up to 6 (AIN) eV. In this case, one of the main requirements is the closeness of the values of lattice constants of neighboring layers, which is necessary in order to prevent both the relaxation of elastic stresses on the interfaces of layers and the formation of misfit dislocations worsening the structural and recombination properties of materials. Reduction of the number of misfit dislocations can be made with the use of graded band-gap structures of multicomponent solid-state solutions in the system AlGaInNPAs.

The use of six-component solid-state solutions enables a more flexible control over the values of lattice constants for the reduction of their misfit between the neighboring layers and allows one, by changing the concentration of reagents in the gas phase or their flows, to grow up graded band-gap structures with the prescribed characteristics in a single technological process [9].

We will use the Weighardt model, in which the values of lattice constants and the energy gap width $E_{g}$ depend linearly on the relative concentrations of binary compounds in the six-component solid-state solution. For the multicomponent system $\operatorname{In}_{x} \mathrm{Ga}_{y} \mathrm{Al}_{1-x-y} \mathrm{~N}_{u} \mathrm{As}_{v} \mathrm{P}_{1-u-v}$, $E_{g}$ and the lattice constant $a$ are calculated in the Weighardt model according to the following expressions:

$$
\begin{aligned}
& E_{g}=x u E_{g}^{\mathrm{InN}}+y u E_{g}^{\mathrm{GaN}}+(1-x-y) u E_{g}^{\mathrm{AlN}}+ \\
& +x v E_{g}^{\mathrm{InAs}}+y v E_{g}^{\mathrm{GaAs}}+(1-x-y) v E_{g}^{\mathrm{AlAs}}+ \\
& +x(1-u-v) E_{g}^{\mathrm{InP}}+y(1-u-v) E_{g}^{\mathrm{GaP}}+ \\
& +(1-x-y)(1-u-v) E_{g}^{\mathrm{AlP}} \\
& a=x u a^{\mathrm{InN}}+y u a^{\mathrm{GaN}}+(1-x-y) u a^{\mathrm{AlN}}+ \\
& +x v a^{\mathrm{InAs}}+y v a^{\mathrm{GaAs}}+(1-x-y) v a^{\mathrm{AlAs}}+ \\
& +x(1-u-v) a^{\mathrm{InP}}+y(1-u-v) a^{\mathrm{GaP}}+ \\
& +(1-x-y)(1-u-v) a^{\mathrm{AlP}}
\end{aligned}
$$

where $E_{g i}$ and $a_{i}$ - the energy gaps and the lattice constants of corresponding binary compounds.

Figure 9 shows the area covered by the sixcomponent solid-state solutions $\operatorname{In}_{x} \mathrm{Ga}_{y} \mathrm{Al}_{1-x-y} \mathrm{~N}_{u} \mathrm{As}_{v} \mathrm{P}_{1-u-v}$ in the coordinates "energy gap width"-"the lattice constant" at the change of the factors $x, y, u$, and $v$ from 0 to 1 , according to calculations by formulas (4) and (5). Vertical lines limit the area of visible radiation (1.5$3 \mathrm{eV})$.

To create biograms in a human visual analyzer, it is necessary that a source of radiation contain the priority wavelengths of $445 \mathrm{~nm}(2.76 \mathrm{eV}), 535(2.29 \mathrm{eV})$, and $570(2.15 \mathrm{eV})$. The corresponding compositions of solidstate solutions are given in Table 1 along with the lattice parameters of perspective substrates (6-10) also are resulted. For these waves, it is desirable to possess a high degree of coherence: $\gamma \approx 0.3-0.5$.

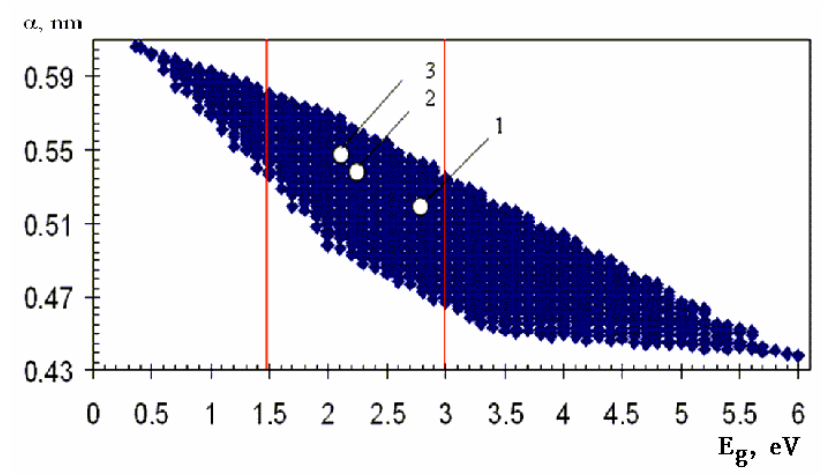

Fig. 9. Optimization of the energy gap and lattice parameters of multicomponent solid-state solutions AlGaInNPAs. Dots 1, 2 , and 3 correspond to the compositions of solid-state solutions given in Table 1. 
In the same way, it is possible to calculate and to produce multispectral layers. To obtain the full spectrum of white light, it is necessary to fabricate a $p$ - $n$ superlattice from $N=\frac{\lambda_{\max }-\lambda_{\min }}{\Delta \lambda}=360 \mathrm{~nm}: 5 \mathrm{~nm}=72 p$ - $n$ heterojunctions (Fig. 10), where $\lambda_{\max }, \lambda_{\min }-$ the red and blue boundaries of the white spectrum (Fig. 11), $\Delta \lambda-$ the width of a single monochromatic spectrum. Some $p-n$ heterojunctions, which are controlled by microprocessors, can radiate a coherent light. For white lasers, the number of $p$ - $n$ heterojunctions should be about 360 . The multicomponent system AlGaInNPAs allows one to produce such structures in MBE or MOCVD epitaxial graded-gap layers [10].

Coherentization of the multispectral radiation of heterogeneous LEDs can occur in open and ring resonators which are formed in separate inhomogeneities of the composition of substances of the active region and injecting layers. They are a system of reflectors, on which there occurs the multiple reflection of light waves, and the certain easily observed angular and axial modes are established. Versions of the open resonators are both a Fabry-Perot resonator with semitransparent mirror and a ring resonator which can include three and more mirrors. In a ring resonator, both running and standing waves can exist, and the last are less energy-favorable and less probable. In superluminescent heterogeneous LEDs [4] under excitation, the channels of many or several open and ring resonators, from which coherent waves go out in various directions, are formed. In fact, such LEDs represent a collection of thresholdless microlasers [11] radiating in various directions. This promotes such a reflection of light from various surfaces of subjects which ensures for the reflected coherent part of the radiation, which have come from various foreshortenings of $3 \mathrm{D}$ patterns, to hit the left and right eyes.

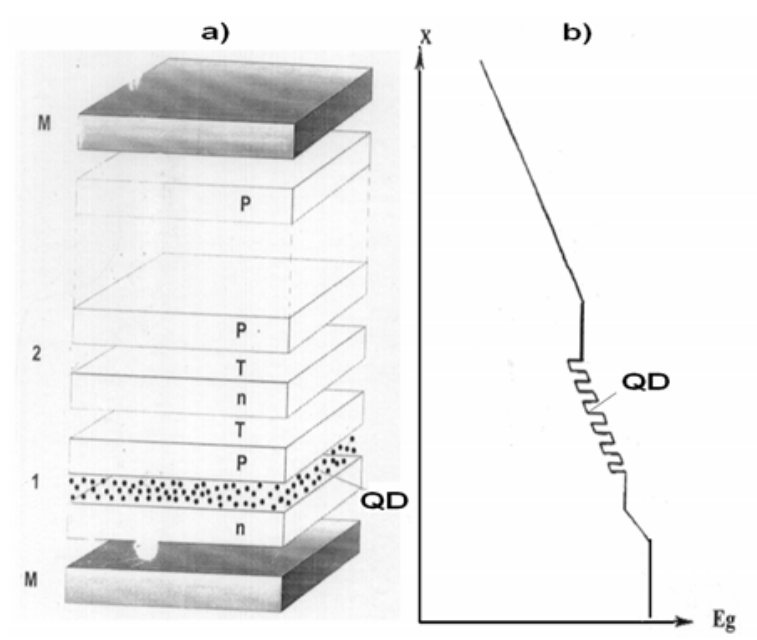

Fig. 10. $a$ - multilayered nanostructures of superluminescent LEDs, $b$ - graded energy gaps with quantum dots (QD) in the multicomponent systems AlGaInNPAs.
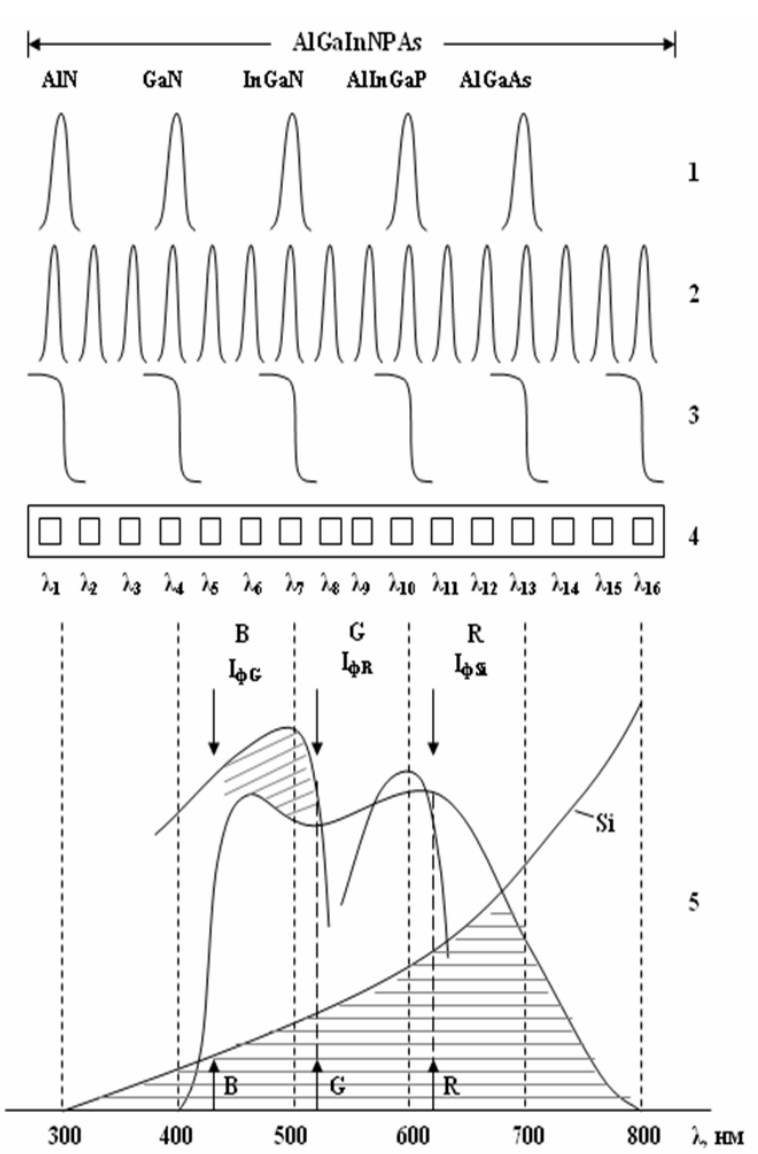

Fig. 11. Multispectral radiation of LEDs (1), lasers (2), and selective sensitivity of photoreceivers (3) created in the multicomponent $\mathrm{A}^{3} \mathrm{~B}^{5}$ systems by energy gap engineering technology; 4 -sequence of LEDs, 5 - white-light spectrum synthesized from $n$ spectra of LED structures and the photosensitivity spectra of $\mathrm{Si}$ and RGB LEDs in the photodiode mode.

\section{Information ability of superluminescent emission of LEDs}

For a LED in the superluminescent mode (Fig. 2d, dashed line 10), the interference pattern is less sharp (less contrast) than that for a laser (Fig. 4 - continuous line 15). The coherence degree is determined to be 0.26 . In the completely spontaneous mode (spectra 1 and 2, Fig. 4), the interference of radiation is very small (continuous line 17, Fig. 4), and the separation of the 1st and 2 nd orders of the interference is impeded. In this case, the coherence degree is smaller than 0.1. This agrees with the classical model of the uptake of light quanta by a photosensitive pigment of retina, rhodopsin, whose number is 15 from 150 quanta hitting eye's cornea at the threshold sensitivity. That is, only the coherent part of a light beam coming to rhodopsin actually creates the variable (dynamic) part of an image (is registered) in layers of rhodopsin.

The important informational consequence of a holographic model of sight is not only a partial 
coherence of light waves, but also its maintenance in reactions with rhodopsin [12]. The time chemical coherence is revealed in both a synchronous course of reactions and a collective transformation of the molecular ensemble which is coordinated by phase. This gives additional opportunities to control the dynamics of changes of undular packages with regard for the new principles of modification of a substance; in our case, it is rhodopsin. In coherent chemistry, chaos becomes order which is the normal state of the system of molecules of a visual analyzer, especially of its higher departments in brain. The chaotic state of the ensemble of molecules represents a pathology. In practice, the control over photochemical reactions in rhodopsin is possible by changing a phase by means of the delay of a light pulse relative to the pulse of response. This allows one to change a state of the ensemble of reacting particles without change of the energy or angular moment. The phototransformation of rhodopsin and its analogs is carried out simultaneously in coherent and incoherent channels [13]. The phototransformation in the incoherent channel represents a statistical thermalization of the excited state and the subsequent slow (2-4 ps) statistical formation of a reaction product. Rhodopsin represents opsin with associated 11-cis-retinal. The coherent transformation consists in the cis-transisomerization of retinal around the $\mathrm{C}(11)=\mathrm{C}(12)$ bond. The direct coherent formation of the first product, bathorhodopsin, from rhodopsin and $h R_{k}$ from halorhodopsin (hR) occurs for $200 \mathrm{fs}$. Moreover, the coherence is maintained in a product. It is established that the coherence degree of rhodopsin is equal to $20 \%$ [12] that is considered as a high level of coherence.

We note that the incoherent part of radiation serves as some catalytic agent of the holographic recording of the information in the ensemble of molecules of rhodopsin. Actually, the information is contained in the coherent part of light waves. This information coherently enters into rhodopsin, by forming biograms which are coherently transmitted to brain in the form of coherent neurograms [13]. The coherent ensembles of molecules of brain carry out the control correlated in time and space of behavior of the whole organism. Further, the coherence is transmitted to ensembles of organisms (the community of people), whose behavior reveals also concerted (correlated) and non-concerted activities analogously to full and partial coherence in optics.

Estimations of the amount of the information at usual three-dimensional vision, which enters into a layer of rhodopsin and then is transmitted by neurons to brain, show that the recording and processing of the information occurs by the principle of the page and block organization of the information stream from all foreshortenings of a three-dimensional image of objects. The volume of information on a page makes $10^{4}-10^{5}$ bits. The number of pages or subholograms (biogrames) in the $x y$ plane of a layer of rhodopsin makes $\left(10^{5}-10^{6}\right)$ spatially separated positions. The full amount of the information which can be written down in two dimensions of a layer of rhodopsin makes $10^{9}-10^{11}$ bits, i.e. of the order of $10^{2}$ Gbits. For the molecular system of rhodopsin, the limiting resolving power makes $10^{7}$ lines $/ \mathrm{mm}$, which corresponds to a density of holographic recording of $10^{15} \mathrm{bits} / \mathrm{cm}^{2}$. At such high resolving power of rhodopsin, the information capacity of holographic records is restricted by the limiting information capacity of a light field at the place of recording and the light field of an illuminated or radiating object, as well as by the biophysical processes running during the photon-electronic (ionic) transformation of signals. We note that the resolving power is evaluated in view of the Rayleigh criteria, according to which the angular uncertainty is $\Delta \alpha=$ $1.22 \lambda / D$ or $\Delta \alpha \approx+\lambda / D$, where $D$ - the diameter of a discrete element of the radiating object. In the real threedimensional structure of molecules of rhodopsin, the three-dimensional recording of biograms and the sampling of neurograms are carried out. In threedimensional holograms, the superposition of interference patterns (subholograms) with one another and their selective reading are realized [14]. In this case, the recording occurs on various wavelengths and under a change of the angle between objective and reference beams. After a transformation of the set of optical subholograms (biograms) into potential reliefs (neurograms), signals become achromatic relative to wavelengths and are supplied in the form of a spectrum of spatial frequencies to the higher departments of the nervous system, where their comparison with and the identification to the signals, which are being stored in the memory, occur. It is known from experience that the phase holograms are registered in three-dimensional holograms, because, at the recording of imposed amplitude holograms, their diffraction efficiency drops proportionally to the square of the number of imposed holograms, which reduces their diffraction efficiency up to inadmissible values. The maximum diffraction efficiency of phase holograms is reached at the equality of the incident angles of the objective and reference light beams, $\Theta_{0}$. With increase in the difference between the incident angles of these beams, the diffraction efficiency decreases down to zero at some critical angle

$\Theta_{0 \mathrm{cr}} \approx \lambda\left(2 \bar{n} h \sin \Theta_{0}\right)$,

where $\bar{n}-$ mean value of the index of refraction, $h-$ the thickness of a layer of rhodopsin, whose optimum value is

$h_{\mathrm{opt}}=\lambda\left(\frac{\Delta n}{\bar{n}}\right)^{-1} \cos \Theta_{R}$,

where $\Delta n \leq 0.001$ - the maximum change of the index of refraction of rhodopsin. At such a change, $\Delta n$, in a crystal of lithium niobate $\mathrm{LiNbO}_{3}$ doped by iron $(\mathrm{Fe})$, it is possible to write down more than 100 holograms into one position by a laser. In order to record 1000 holograms into one position, it is required to change the 
incident angle of the reference beam by $8-10^{\circ}$. The experimentally established diffraction efficiency of three-dimensional phase holograms of the mentioned type is $20-30 \%$. The imposed phase holograms unlike the amplitude ones change slightly their diffraction efficiency, which admits the superposition of several hundreds of holograms. In the above-mentioned experiment with lithium niobate at the superposition of 512 holograms, the diffraction efficiency has changed from 25 down to $2 \%$. The last value is sufficient for the conformation transformation of rhodopsin which gives the necessary threshold value of neuron potential. Hence, it is possible to write down more than 100 foreshortenings in a single three-dimensional volume of rhodopsin without mutual interferences of the first order. Then the total amount of the filed information reaches a value of $10^{13}$ bits, which is in agreement with the numbers of neurons and links between them, $10^{12}-10^{13}$, in the higher departments of the nervous system.

These estimations allow one to refine the requirements to information parameters of the radiation of superluminescent LEDs, in which it is important to have a coherence degree within the limits of not less than $0.1-0.5$ and the angular orientation in the given interval of wavelengths of at least $10^{\circ}$ in addition to the requirements to the intensity of light, the spectrum of radiation, luminosity, power of consumption, speed, and energy efficiency.

The amount of the information about the external world, which is perceived by visual analyzers in the form of reflected or emitted radiation, is determined by the number of variants of the registered holograms and biograms in each of them and also by the probability of their transformation in neurograms in a space-frequency representation of their spectrum [15]. According to the Kotelnikov-Shannon theorem, the amount of the information transmitted along one channel is

$I_{i}=C S_{0} \log _{2}\left[1+\frac{k_{i}^{2}}{k_{\mathrm{thr} . i}^{2}}\right] \Delta \omega$,

where $k_{i}$ - contrast of the spatial harmonic $i$ in the image of an object against the background; $k_{\mathrm{thr} . i}-$ threshold contrast at the distinction of a dashed object by eye at a frequency $\omega_{i}$ and with a probability of $50 \%$ which depends on noise, $S_{0}$ - the area of the image of an object, $\Delta \omega-$ a band of spatial frequencies, and $C-$ a constant.

The total amount of information which enters the higher nervous departments of a human brain is

$I=\sum_{i} C S_{0} \log \left[1+\frac{k_{i}^{2}}{k_{\mathrm{thr} . i}^{2}}\right] \Delta \omega$.

In addition to purely optical effects (interference, gains, etc.), electromagnetic waves have losses of information at a radiative transfer in biological media of the visual system. There occurs a distortion of the structure of electromagnetic waves which is determined by the interaction of a wave with physical inhomogeneities of the medium. For the analysis of the optical channels present in such media, it is possible to use the approach developed for systems of optical communication [16]. During the propagation, optical signals are absorbed and scattered, which causes the amplitude and spatial changes, whose random character is described in the theory of optical communication by field coherence functions. The solution of a wave equation by methods of perturbation theory shows that the normalized amplification of electromagnetic waves obeys the logarithmic normal distribution [15, 16]. Because the attenuation in a communication channel determines the volume of the information transferred by it, we can write

$I_{0}=A e^{k}$,

where $k$ - contrast at incoherent light or the degree of coherence at partially coherent light, and $A$ normalizing factor.

It is logical to assume that, with increase in the degree of coherence $\gamma$, the contrast of the image $K$ also increases, which causes a growth of the information ability of partially coherent radiation.

Two models of holographic perception of images are possible: 1 - the information is contained only in the coherent part of a light beam, and the incoherent radiation is a distinctive catalytic agent of the recording of biograms, 2 - the information is contained both in the coherent and incoherent components of a light beam. In such an approach, the coherent part is characterized by a coherence degree, and the incoherent one by the contrast $K$. By analogy to the threshold contrast, we introduce a threshold coherence degree $\gamma_{\mathrm{thr}}$, at which the electrical potential in rhodopsin excited by the coherent component of a LED radiation is equal to the mean squared amplitude of fluctuations in the biomedium of rhodopsin-neuron contacts.

According to the more general second model, the information capacity of man's visual system is as follows:

$$
\begin{aligned}
& I=I_{\mathrm{coh}}+I_{\mathrm{incoh}}-I_{0}=\sum_{i} C S \log _{2}\left(1+\frac{\gamma^{2}}{\gamma_{\mathrm{thr}}^{2}}\right) \Delta \omega+ \\
& +\sum_{i} C S \log _{2}\left(1+\frac{k^{2}}{k_{\mathrm{thr}}^{2}}\right) \Delta \omega-A e^{k}= \\
& =\sum_{i} C S \Delta \omega \log _{2} \frac{2 \gamma^{2}\left(k^{2}+k_{\mathrm{thr}}^{2}\right)}{\gamma_{\mathrm{thr}}^{2} k_{\mathrm{thr}}^{2}}-A e^{k},
\end{aligned}
$$

where $I_{0}=H_{0}$ - the decrease of the information determined by the uncertainty (i.e. by entropy) created by fluctuations and the absorption of electromagnetic waves because of the inhomogeneities of elements of man's visual system: pupil, retina, rods, cones, ellipsoids, rhodopsin, neurons, and brain cells. This value of entropy 
increases with the degrees of coherence and contrast by the exponential law $H_{0}=A e^{K}$ (Fig. 12, curve 5), where $A$ - a constant of the visual apparatus. At great $\gamma$ and $K$, this leads to a replacement of the dependence $I=f(\gamma, k)$ by the opposite one - the information capacity achieves a maximum and then drops.

Since the information ability of the coherent component exceeds that of the incoherent component by many times, it is natural to assume that the recording of coherent and incoherent radiation occurs mainly, respectively, in cones and rods. Theoretically, the purely laser coherent radiation allows one to register a maximum amount of information (Fig. 12, dotted curve 1). Really, the diminution of a share of incoherent radiation with increase in $\gamma(\gamma>0.5)$ leads to weakening the role of catalytic and biophysical mechanisms of maintenance of the processes running in rhodopsin and the neuro- and molecular system of brain which are a subject to be refined. This leads to a deceleration of the growth of the information capacity, and then its diminution with increase in the degree of coherence (Fig. 12, solid curve 2). If the visual apparatus perceives only the incoherent radiation and holograms are not formed, then the information capacity increases practically linearly with the contrast $k / k_{\text {thr }}$ (curve 3, Fig. 12). However, the absolute value of the information capacity is less by $3-5$ orders. The last variant corresponds to the radio engineering model of visual system [8] which is widely applied to the systems of TV, robotics, etc.

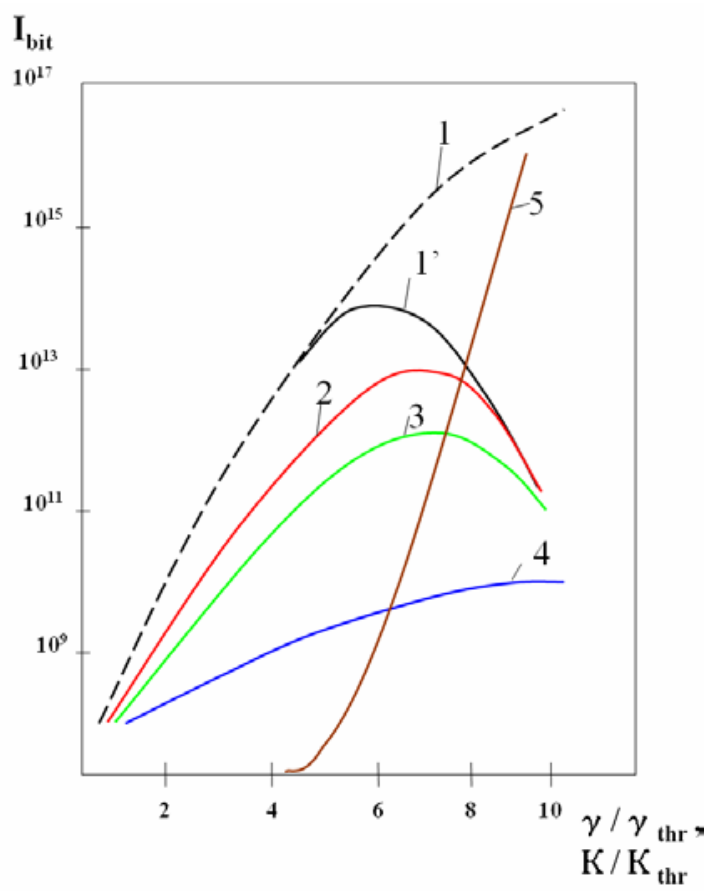

Fig. 12. Information ability of a superluminescent LED as a function of the degree of coherence $\gamma / \gamma_{\mathrm{thr}}$ and the contrast $k / k_{\mathrm{thr}}$ : laser coherent radiation $\left(1, l^{\prime}\right)$; partially coherent radiation at $J=500 \mathrm{~A} / \mathrm{cm}^{2}$ (2) and $100 \mathrm{~A} / \mathrm{cm}^{2}$ (3); noncoherent light at $700 \mathrm{~A} / \mathrm{cm}^{2}$ (4).
At $S=1 \mathrm{~mm}^{2}$, we obtain $I=2.510^{9}$ bits for one information channel, which agrees with the information ability of man's visual apparatus [1].

In Fig. 12, curves 1, 2, and 3 are obtained on the basis of our experimental data for a LED with the set of quantum points in a heterogeneous material of the system InGaAsP at various levels of excitation (Fig. 4). Selective epitaxy of $\mathrm{A}^{3} \mathrm{~B}^{5}$ compounds allow one to produce $\mathrm{Si} / \mathrm{A}^{3} \mathrm{~B}^{5}$ integrated circuits with high-quality selective areas without dislocations (Fig. 13a).

The consideration of the conception of perception of images at solid-state lighting allow us to use methods of optical communication for the optimization of systems of registration of images at the illumination by diodes [16].

The microprocessor integrated with a matrix of LEDs drives the integral elements of switching (transistors) by signals of the photodetectors with feedback which are built in the integrated circuit (Fig. 13b). Since the speeds of LEDs, switchboards, and the microprocessor exceed the speed of a human visual analyzer by several orders, the dynamic control of the process of illumination, which is perceived by eyes as a stationary one, is realized. The control can be carried out both by the program of a microprocessor and by the operator with the use of a panel. In the development and the operation of open diode illumination circuits, it is necessary to consider the influence of effects of turbulence, scattering, and fading in the atmosphere which accompany the open systems of communication. In optical fibers of cones and rods, there occur the spatial digitization of the interference patterns of electromagnetic waves and the separation of their mode structure which enters the ellipsoid representing an analog of a lens with refractive index variable over a cross-section (a gradan). The modeling and analysis of the passage of radiation through the fiber gradans of rods and cones with the use of methods developed in the optics of quartz fibers allows one to optimize both the heterostructure of the matrix of LEDs and the program of the microprocessor-based control over its elements.

The above-considered model of perception and processing of images in man's visual analyzer includes the spatial digitization of input optical signals, their fast Fourier transformation, and the formation of holograms (biograms), the receptor potentials (neurograms) from which carry out the recognition and the identification of 3D images in man's brain [17].

As the process of identification and processing of images is dynamic, the biograms are registered in man's memory, where they are compared with the available information. The optoelectronic system of registration of biograms includes light-emitting diode sources of illumination, photosensitive elements (control sensors) in the corresponding spectral subranges of illumination and a background, logic and remembering elements in the structure of a programmed microprocessor, and the elements of feedback and switching. 
a)

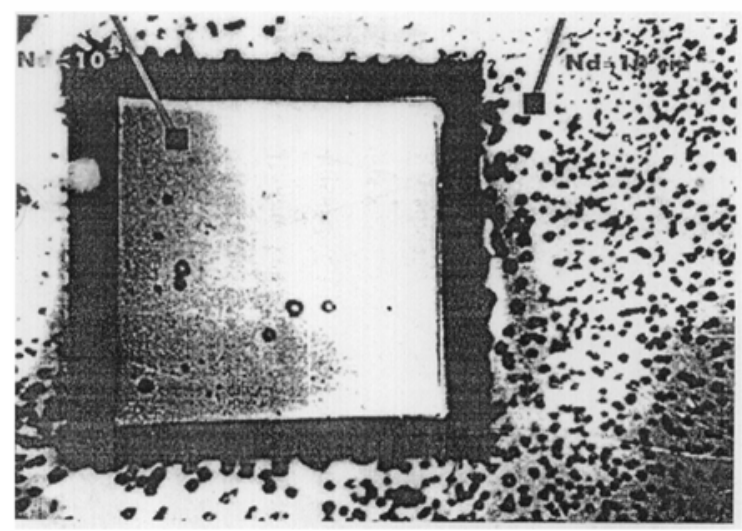

b)

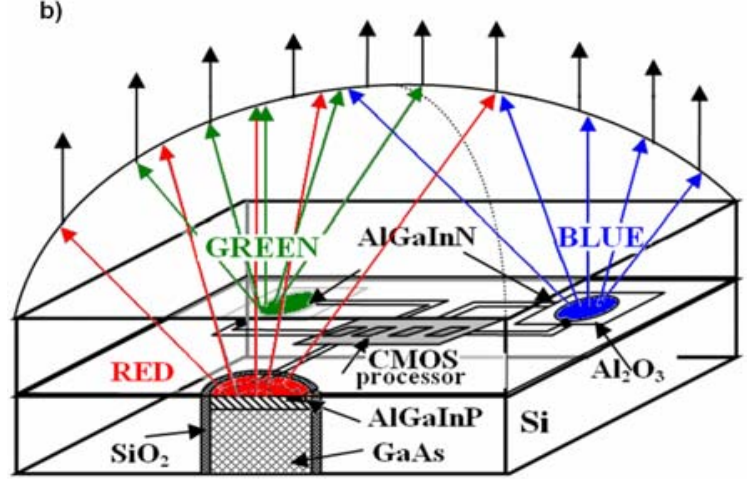

c)

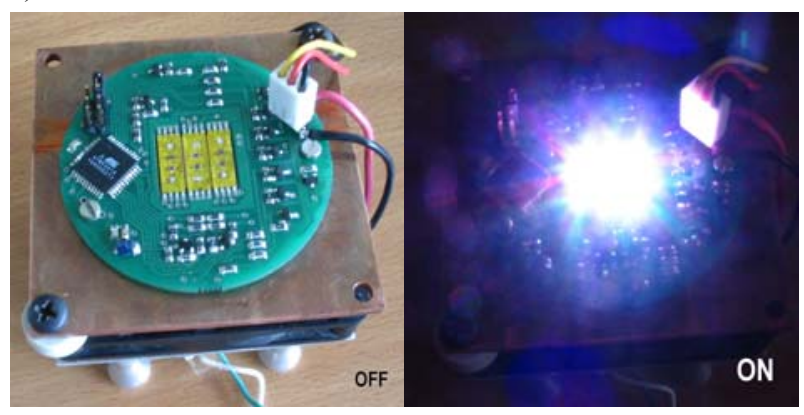

Fig. 13. $a$ - Si-based integral circuit with dislocationless GaAs grown by vapor phase epitaxy in selective regions as a substrate for LEDs [18]; $b-$ one-chip $\mathrm{Si} / \mathrm{A}^{3} \mathrm{~B}^{5}$ SSL optoelectronic processor; $c-$ photohybrid $\mathrm{Si} / \mathrm{A}^{3} \mathrm{~B}^{5} \mathrm{SSL}$ optoelectronic processor in the "off" and "on" modes.

Programming the microprocessor should be carried out in view of both the formation of biogramsneurograms in the visual analyzer and their dynamics under the light-emitting diode illumination for the optimum perception of images.

For an information-technological realization of diode light sources, it is expedient to create the epitaxial multispectral multilayered nanostructures on the basis of multicomponent solid-state solutions of compounds $\mathrm{A}^{3} \mathrm{~B}^{5}$ which are integrated in a hybrid or monolithic way with $\mathrm{Si} / \mathrm{GaAs}$ transistor microprocessors, control sensors, and microoptics (Fig. 13b).

The optimization of the perception of images by an optoelectronic microprocessor at the diode illumination allows one to perceive the information equivalent to that created at the power efficiency of traditional sources of illumination of not less than $150-200 \mathrm{Lm} / \mathrm{W}$, at which the transition from the lamp lighting to the solid-state one is economically expedient (see Table 2 ).

\section{Application of the holographic model of sight in medicine}

Lasers and LEDs are widely used for prophylaxis and therapy of various diseases, especially those concerning sight. In this case, specific features of the influence of the coherent and incoherent components of electromagnetic radiation are not considered. As usual, one chooses the power of a light beam and its wavelength as the main parameters of radiation which define its influence on organisms. At the same time, the partial coherence of radiation of superluminescent lightemitting diodes corresponds to the solar radiation and the radiation reflected from objects and is the closest analog of natural illumination which is available in the nature and to which alive organisms have adapted during their evolution.

With regard for the partial coherence of radiation, it is expedient to consider two aspects in medical practice: the informational and energetic components of the influence of light on organisms. The first component influences, through cerebral cortex, the whole organism and its parts by means of the cybernetic control over their functioning. The second, energetic effect of light is local. It allows one to measure the structure or composition of molecules of an alive organism, which is widely used in modern photon medicine.

From practice, separate informational techniques of treatment and prophylaxis of diseases are known. However, they bear a spontaneous, sometimes mysterious, character and are not based on a scientifically proved methodology. In most cases, the informational technologies of treatment are realized not through man's visual apparatus, through which more than $90 \%$ of the information is supplied, but through the less informative sense organs - hearing, touch, scent. Using the modern microprocessor technique will allow one to purposefully introduce the preset information flows in brain through the partially coherent radiation of superluminescent light-emitting diodes. Such flows will "adjust" an organism on the eradication of undesirable changes in it which cause diseases (Table 2). Practically, the microprocessor will control not only the intensity and the spectral structure, but also the degree of coherence, polarization, and the other parameters of radiation. In addition to the perspectives of applications in medicine, the above-considered holographic model opens tempting prospects in the field of training, sports, cultures, and in the other fields of activity of the Man. 
Table 2. Parameters of models of sight and LED-based sources for medicine and common lighting applications.

\begin{tabular}{|l|l|c|c|c|c|c|c|c|c|}
\hline & \multicolumn{1}{|c|}{$\begin{array}{c}\text { Models } \\
\text { Parameters }\end{array}$} & $\begin{array}{c}1 \mathrm{D} \\
\text { (Radio) }\end{array}$ & $\begin{array}{c}\text { 3D } \\
\text { Holographic }\end{array}$ & Lamps & $\begin{array}{c}\text { LED }+ \\
\text { Phosphor }\end{array}$ & $\begin{array}{c}\text { RGB LED } \\
\text { matrix }\end{array}$ & $\begin{array}{c}\text { RGB } \\
\text { Partially } \\
\text { coherent } \\
\text { matrix }\end{array}$ & Medicine & $\begin{array}{c}\text { Lomm. } \\
\text { lighting }\end{array}$ \\
\hline 1. & $\begin{array}{l}\text { Information } \\
\text { capacity, bit }\end{array}$ & $10^{6}$ & $5 \cdot 10^{13}$ & $10^{6}$ & $10^{6}$ & $10^{8}$ & $5 \cdot 10^{13}$ & $5 \cdot 10^{13}$ & $10^{13}$ \\
\hline 2. & $\begin{array}{l}\text { Information } \\
\text { rate of sight, } \\
\text { bits/s }\end{array}$ & 70 & $16 \cdot 10^{6}$ & 70 & 70 & 103 & $16 \cdot 10^{6}$ & $16 \cdot 10^{6}$ & $16^{\circ} \cdot 10^{6}$ \\
\hline 3. & $\begin{array}{l}\text { Resolving } \\
\text { power, } \\
\text { lines/mm }\end{array}$ & $10^{4}$ & $10^{7}$ & $10^{4}$ & $10^{4}$ & $10^{7}$ & $10^{7}$ & $10^{7}$ & $10^{5}$ \\
\hline 4. & Sight speed, s & $10^{-1}$ & $10^{-4}$ & $10^{-1}$ & $10^{-1}$ & $10^{-4}$ & $10^{-5}$ & $10^{-9}$ & $10^{-5}$ \\
\hline 5. & $\begin{array}{l}\text { Informative } \\
\text { efficiency } \\
\text { bit/Lm }\end{array}$ & 20 & $10^{3}$ & 20 & 20 & $10^{3}$ & $10^{5}$ & $10^{4}$ \\
\hline 6. & $\begin{array}{l}\text { Energetic } \\
\text { efficiency, } \\
\text { Lm/W }\end{array}$ & 60 & 200 & $1-20$ & 20 & 60 & 60 & 60 & $>200$ \\
\hline 7. & Life time, h & $10^{5}$ & $10^{5}$ & $10^{2}$ & $10^{2}$ & $10^{5}$ & $10^{5}$ & $10^{5}$ & $10^{5}$ \\
\hline 8. & $\begin{array}{l}\text { Specific cost, } \\
\text { \$/Lm }\end{array}$ & $10^{-3}$ & $10^{-5}$ & $10^{-3}$ & $10^{-2}$ & $10^{-2}$ & $10^{-4}$ & $10^{-4}$ & $10^{-5}$ \\
\hline 9. & $\begin{array}{l}\text { Expenditure, } \\
\text { arb. units }\end{array}$ & 10 & 5 & 100 & 7 & 6 & 10 & 30 & 1 \\
\hline
\end{tabular}

\section{Conclusions}

1. For the first time, we have determined the properties of partially coherent radiation and presented the requirements following from them to the heterostructures of superbright LEDs and to their modes of excitation and functioning in the information system "LED illumination - man's visual analyzer".

2. A phenomenological model of perception of the information by a man at the illumination of objects by the partially coherent radiation of superluminescent LEDs is proposed. It is shown that the ability of perception of the information increases with the degree of coherence $\gamma$ up to 0.5 . In the case where $\gamma>0.5$, there occurs a reduction of the information capacity due to the increase of entropy in biophysical communication channels of man's visual analyzer.

3. The most perspective structures of superluminescent LEDs are devices with heterogeneous active area, whose heterogeneity is represented by microdomains of resonators (microcavities). They are semilasers.

4. For the first time, the attention is focused on various roles of the coherent and incoherent components of the partially coherent radiation of superluminescent LEDs, the coherent character of photochemical transformations of rhodopsin, and the subsequent coherent transfer of the information to brain. In this case, the incoherent component serves as a catalyst of both the holographic recording of the information in the ensemble of rhodopsin molecules and other biochemical processes.

The decisive role of the coherent component in the processes of reception of the information by a man through light waves represents the general law of the nature on a higher stability of the ranked systems in comparison with chaotic ones. The partial coherence is a part of this law.

We expect that the most perspective region of applications of the holographic model of sight will be the treatment of various diseases of men/women, especially those of sight, with the use of LED-based lighting.

\section{References}

1. V.I. Osinsky, Optimization of LED nanostructures at solid state lighting // Electronics and Communications, Special issue "Electronics Problems", p. 21-28 (2006).

2. V.I. Osinsky, V.I. Privalov, O.Ya. Tikhonenko, Optoelectronic Structures on Multicomponent Semiconductors. Nauka i Tekhnika, Minsk, 1981 (in Russian).

3. Yu.A. Vladimirov, D.I. Roschunkin, A.Ya. Potapenko et al., Biophysics. Meditsina, Moscow, 1983 (in Russian).

4. V.I. Osinsky, Optoelectronic properties of heterogeneous semiconductors at superhigh time and space frequencies // Proc. 5-th Intern. Symposium on Recent Advances in Microwave Technology. Kyiv, Sept. 11-16, 1995, p. 433-436.

5. Sol. P. Dijaili, J.M. Wachsman, A comparison of chipbased optical amplifiers // Laser Focus World 39, No 6, p.145-148 (2003).

6. G.S. Landsberg, Optics. Nauka, Moscow, 1976.

7. V.K. Albekov, P.I. Zubkov and A.V. Frolov, Optical and Optoelectronic Processing of 
Information. Mashinostroenie, Moscow, 1976 (in Russian).

8. N.N. Krasilnikov, Theory of Transfer and Perception of Images. Radio i Svyaz, Moscow, 1986 (in Russian).

9. V.G. Verbitsky, S.V. Osinsky, A.V. Saricov, The calculation of elemental concentration of $\mathrm{A}^{3} \mathrm{~B}^{5}$ graded band structures for white LEDs // Phys. Surf. Eng. 1, No. 3-4, p. 341-346 (2003).

10. V.G. Verbitsky, V.I. Osinsky, Yu.G. Mokeev, O.F. Nemchin et al., White superluminescent LED and the method of its production // Patent of Ukraine, \#56544 A, 17.07.2002.

11. H. Yokoyama, Physics and Device Applications of Optical Microcavities, in: Confined Electrons and Photons: New Physics and Applications, Eds. E. Burstein and C. Weisbuch. Plenum Press, New York, 1995, p. 865-868.

12. M.A. Ostrovsky, Molecular mechanism of cracking light action on eye structures and protection systems from such cracking // Adv. Biol. Chem. 45, No. 3, p. 173-204 (2005).
13. T. Kakitani, R. Akiyama, Y. Hatano et al. // J. Phys. Chem. B. 102, p. 1334 (1998).

14. Yu.N. Denisyuk, Foundations of Holography. Vavilov SOI, Leningrad, 1978 (in Russian).

15. V.V. Mikhnevich, V.A. Grigor'ev, V.F. Koshchavtsev, D.S. Sokolov, Solid State Image Transformers, Ed. V.I. Osinsky. Nauka i Tekhnika, Minsk, 1980 (in Russian).

16. V.I. Osinsky, Application of optical communication to the systems of image registration at diode lighting // Proc. Conference on Radio, Telecommunication and Information Technologies, Zaporizhzhya, April, 2006, p. 10-11.

17. V.I. Osinsky, Information ability of human perception of partially coherent radiation of superluminescent LEDs // Electronics and Communications 3 (32), p. 13-21 (2006).

18. 18. V.I. Osinsky, P.F. Olexenko, A.V. Palagin, V.G. Verbitsky et al., The problems of integration of heteroelectronic structures with silicon integrated circuits // Konstruirovaniye Tekhnologiya Radioapparatury 1 (1), p. 1-17 (1999) (in Russian). 